\title{
Building a Large Syntactically-Annotated Corpus of Vietnamese
}

\author{
Phuong-Thai Nguyen \\ College of Technology, VNU \\ thainp@vnu.edu.vn \\ Xuan-Luong Vu \\ Vietnam Lexicography Cen- \\ tre \\ vuluong@vietlex.vn
}

\author{
Thi-Minh-Huyen Nguyen \\ University of Natural \\ Sciences, VNU \\ huyenntm@vnu .edu.vn
}

\author{
Van-Hiep Nguyen \\ University of Social Sciences and \\ Humanities, VNU \\ hiepnv@vnu.edu.vn
}

\section{Introduction}

Treebanks are used for training syntactic parsers, part-of-speech taggers, and word segmenters. These systems then can be used for applications such as information extraction, machine translation, question answering, and text summarization. Treebanks are also useful for linguistic studies, for example the extraction of syntactic patterns or the investigation of linguistic phenomena. Recently, treebanks and other large corpora have become more important since the development of powerful machine learning methods.

As mentioned above, Vietnamese is an isolating language. There is no word delimiter in Vietnamese. The smallest unit in the construction of words is syllables. Words can be single or compound. Vietnamese script is invented based on

\author{
Hong-Phuong Le \\ LORIA/INRIA Lorraine \\ lehong@loria.fr
}

Latin alphabet in which the expansion includes accent characters and stressed accents.

Since Vietnamese word order is quite fixed, we choose to use constituency representation of syntactic structures. For languages with freer word order such as Japanese or Czech, dependency representation is more suitable. We apply annotation scheme proposed by Marcus et al. (1993). This approach has been successfully applied to a number of languages such as English, Chinese, Arabic, etc.

For Vietnamese, there are three annotation levels including word segmentation, POS tagging, and syntactic labeling. Word segmentation identifies word boundary in sentences. POS tagging assigns correct POS tags to words. Syntactic labeling recognizes both phrase-structure tags and functional tags. Our main target is to build a corpus of 10,000 syntactically-annotated sentences (trees) and an additional POS tagged data set of 10,000 sentences. Treebank construction is a very complicated task including major phases: investigation, guideline preparation, building tools, raw text collection, and annotation. This is a repeated process involving especially three phases: annotation, guideline revision, and tool upgrade. Raw texts are collected from a newspaper source, the Youth online daily newspaper, with a number of topics including social and politics. We completed about 9,500 trees and 10,000 POS tagged sentences.

In order to deal with ambiguities occurring at various levels of annotation, we systematically applied linguistic analysis techniques such as deletion, insertion, substitution, questioning, transformation, etc. Notions for analysis techniques are described in guideline. These techniques are originated in literatures or proposed 
by our group. They are described with examples, arguments, and alternatives. For automatic labeling tools, we used advanced machine learning methods such as CRFs for POS tagging or LPCFGs for syntactic parsing. These tools helped us speed up labeling process. Besides, tree editor was also very helpful.

Our treebank project is a branch project of a national project which aims to develop basic resources and tools for Vietnamese language and speech processing. This national project is called VLSP $^{1}$. In addition to treebank, other textprocessing resources and tools include: Vietnamese machine readable dictionary, EnglishVietnamese parallel corpus, word segmenter, POS tagger, chunker, and parser. Treebank and tools are closely related. Tools are trained using treebank data, and then they can be used in treebank construction.

The rest of this paper is organized as follow: First, we present issues in Vietnamese word segmentation problem. Second, POS tagging and syntactic parsing are described. Third, tools and annotation process are represented. Fourth, we present annotation agreement evaluation. And last, some conclusion is drawn.

\section{Word Segmentation}

There are many approaches to word definition, for example based on morphology, based on syntax, based on semantics, or linguistic comparison. We consider words as syntactic atoms (Sciullo and Williams, 1987) according to the sense that it is impossible to analyze word structure using syntactic rules, or that words are the smallest unit which is syntactically independent. We choose this criterion partly because the first application of word segmentation is for syntactic analysis (build trees).

According to application view, machine translation researchers may argue that Vietnamese words and foreign words should match each other. The problem is that there are so many possible foreign languages which are different in vocabulary. Dictionary editors may want to extract phrases from text which need to be explained in meaning. For this application, syntactic parsers can be used as tool for editors. Parsers can extract candidates for phrase/word entry.

The following word types are considered in word segmentation phase: single words, compound words, repeated words, idioms, proper

\footnotetext{
${ }^{1}$ Vietnamese Language and Speech Processing
}

names, date/time, number expressions, foreign words, abbreviations.

Word segmentation ambiguity is the major problem annotators have to deal with. Suppose that three words "nhà cửa", "sắc đẹp", and "hiệu sách" are being considered. Annotators need to identify these combinations as words in:
a. Nhà cửa bề bộn quá
b. Cô ấy giữ gìn sắc đẹp.
c. Ngoài hiệu sách có bán cuốn này

And not words in:

a. Ở nhà cửa ngõ chẳng đóng gì cả.

b. Bức này màu sắc đẹp hơn.

c. Ngoài cửa hiệu sách báo bày la liệt.

We used dictionaries as a reference. In practice, we consider dictionary words as candidate for word segmentation and make decision using context.

\section{POS Tagging and Syntactic Annota- tion Guidelines}

\subsection{POS Tag Set}

For European languages, word classes closely relate to morphological aspects such as gender, number, case, etc. For Vietnamese, words are often classified based on their combination ability, their syntactic functions, and their general meaning. We choose first two criteria, combination ability and syntactic function, for POS tag set design. Therefore our POS tag set will not contain morphological information (number, aspect, tense, etc.), sub-categorization information (transitive/intransitive verbs, verbs followed by clauses, etc.), and semantic information.

\subsection{Syntactic Tag Set}

Our tag set contains three tag types: constituency tags, functional tags, and null-element tags. We use the tag $\mathrm{H}$ to label phrase head. If a phrase has more than one head, connected by coordination conjunctions or commas, then all heads are labeled with $\mathrm{H}$ tag. Other treebanks often does not use head tag. Therefore researchers on syntactic parsing (Collins, 1999) used heuristic rules to determine CFG rules' head. Machine learning methods also can be used (Chiang and Bikel, 2002). Null elements are often used for adjective clauses, ellipsis, passive voice, and topic.

\subsection{Sentence and Phrase Analysis Tech- niques}

Annotation of real text requires various techniques to be applied. Ambiguity may occur in many steps of analysis such as determining 
phrase's head, discriminating between possible complements, discriminating between adjuncts and other sentence elements, etc. Sentence analysis techniques include deletion, substitution, insertion, transformation, questioning. These techniques exploit contextual information, word combination, word order, and functional words to disambiguation between possible structures.

\subsection{Linguistics Issues}

The problem of treebank construction can be considered as an application of linguistic theories though treebanks can also be used for linguistic studies. However, there are still disagreements among linguists as to solutions for many linguistic issues. For example, that the classifier noun is noun phrase's head or pre-modifier is controversial. Another example, Vietnamese sentence structure is subject-predicate or topic-comment is also controversial. Our treebank relies more on subject-predicate structure. Moreover, we choose linguistic solutions most appropriate to our design.

\section{Tools}

We designed a tool for supporting annotators in most all phases of the annotation process. Main functions of our editor are as follows:

- Edit and view trees in both text mode and graphical mode

- View log files, highlight modifications

- Search by words or syntactic patterns

- Predict errors (edit, spell, or syntax)

- Compute annotation agreement and highlight differences

- Compute several kinds of statistics

For encoding the treebank, we have developed an exchange format named vnSynAF, a syntactic annotation framework which is conformed to the standard framework SynAF of ISO. The framework SynAF is built on top of an XML-based annotation scheme which is recommended by ISO for the encoding of treebanks ${ }^{2}$. Our tool also supports bracketing representation (or Lisp style) of Penn English Treebank. These formats can be converted into each other.

For the task of word segmentation, we used vnTokenizer, a highly accurate segmenter which uses a hybrid approach to automatically tokenize Vietnamese text. The approach combines both finite-state automata technique, regular expres- sion parsing, and the maximal-matching strategy which is augmented by statistical methods to resolve ambiguities of segmentation (Phuong et al., 2008).

We used JVnTagger, a POS tagger based on Conditional Random Fields (Lafferty et al., 2001) and Maximum Entropy (Berger et al., 1996). This tagger is also developed under supported of VLSP project. Training data size is 10,000 sentences. Experiments with 5-fold cross validation showed that $\mathrm{F} 1$ scores for CRFs and Maxent are $90.40 \%$ and $91.03 \%$ respectively.

A syntactic parser based on Lexicalized Probabilistic Context-free Grammars (LPCFGs) is another tool we used. Another group in VLSP customized Bikel's parser ${ }^{3}$ for parsing Vietnamese text. This parser is a well designed and easy to adapt to new languages. The group implemented a Vietnamese language package which handles treebank, training, finding head of CFG rules, and word features. This parser can output text with constituent tags only or both constituent tags and functional tags.

\section{Annotation Process and Agreement}

There are three annotation levels: word segmentation, POS tagging, and syntactic labeling. Since the word segmentation tool had been available before the start of our project, it was used for the first annotation level (word segmentation) immediately. As to the other annotation levels (POS tagging and syntactic parsing), first several thousand sentences were labeled manually. After that a POS tagger and a parser are trained bimonthly, then the annotation task becomes semiautomatic. According to our annotation process, each sentence is annotated and revised by at least two annotators. The first annotator labels raw sentences or revises automatically-analyzed sentences. Then the second annotator revises the output of the first annotator. In addition, we also check corpus by syntactic phenomena, for example direction words, questions, etc. This process is supported by tool. So there are many sentences which are revised more than twice.

Table 2 shows a number of important corpus statistics such as sentence count, word count, and syllable count for two data sets. We completed the POS tagged data set and will complete the syntactically-labeled data set soon. The average sentence length is about 21.6 words.

\footnotetext{
${ }^{2}$ ISO/CD/24615, Language Resource ManagementSyntactic Annotation Framework (SynAF) TC37/SC 4 N421, 22th Aug 2007, http://tc37sc4.org/documents
}

\footnotetext{
${ }^{3}$ http://www.cis.upenn.edu/ d dbikel/software.html
} 


\begin{tabular}{|l|l|l|l|}
\hline Data set & Sentences & Words & Syllables \\
\hline POS tagged & 10,368 & 210,393 & 255,237 \\
\hline $\begin{array}{l}\text { Syntactically } \\
\text { labeled }\end{array}$ & 9,633 & 208,406 & 251,696 \\
\hline
\end{tabular}

Table 1. Corpus statistics

Annotation agreement measures how similar two texts annotated independently by different annotators are. Since this problem is similar to parsing evaluation, we use parseval measure. First, syntactic constituents in the form (i, j, label) are extracted from syntactic trees. Then tree comparison problem is transformed into constituent comparison. We can compute three kinds of measurement: constituent and function similarity, constituent similarity, and bracket similarity. By using this method, we can evaluate both overall agreement and constituency agreement.

Annotation agreement A between two annotators can be computed as follows:

$$
A=\frac{2 \times C}{C_{1}+C_{2}}
$$

where $C_{1}$ is the number of constituents in the first annotator's data set, $\mathrm{C}_{2}$ is the number of constituents in the second annotator's data set, and $\mathrm{C}$ is the number of identical constituents. Table 3 shows an example of constituent extraction from trees. From Table 3, we can compute: $\mathrm{C}_{1}=6 ; \mathrm{C}_{2}=7 ; \mathrm{C}=6 ; \mathrm{A}=12 / 13=0.92$.

\begin{tabular}{|c|c|}
\hline $1^{\text {st }}$ annotator & $2^{\text {nd }}$ annotator \\
\hline $\begin{array}{l}\text { (S (NP (Np Hằng)) } \\
\text { (VP (V ngắm) } \\
\text { (NP (N mưa)) } \\
\text { (PP (E trong) } \\
\quad(\mathrm{NP} \quad(\mathrm{N} \text { công } \\
\begin{array}{l}\text { viên))) }) \\
(. .))\end{array}\end{array}$ & $\begin{array}{l}\text { (S (NP (Np Hằng)) } \\
\text { (VP (V ngắm) } \\
(\mathrm{NP}(\mathrm{NP}(\mathrm{N} \text { mưa })) \\
(\mathrm{PP}(\mathrm{E} \text { trong) } \\
\quad(\mathrm{NP} \\
\text { công viên))))) } \\
(\mathrm{N}\end{array}$ \\
\hline $\begin{array}{l}(1,6, \mathrm{~S}) ;(1,1, \mathrm{NP}) ;(2,5, \mathrm{VP}) \\
(3,3, \mathrm{NP}) ;(4,5, \mathrm{PP}) ;(5,5, \mathrm{NP})\end{array}$ & $\begin{array}{l}(1,6, \mathrm{~S}) ;(1,1, \mathrm{NP}) ;(2,5, \mathrm{VP}) \\
(3,3, \mathrm{NP}) ; \quad(3,5, N P) ; \quad(4,5, \\
\mathrm{PP}) ;(5,5, \mathrm{NP})\end{array}$ \\
\hline
\end{tabular}

Table 2. Constituent extraction from trees

We carried out an experiment involving 3 annotators. They annotated 100 sentences and the result is shown in Table 4.

\begin{tabular}{|l|l|l|l|}
\hline Test & A1-A2 & A2-A3 & A3-A1 \\
\hline Full tags & $90.32 \%$ & $91.26 \%$ & $90.71 \%$ \\
\hline $\begin{array}{l}\text { Constituent } \\
\text { tags }\end{array}$ & $92.40 \%$ & $93.57 \%$ & $91.92 \%$ \\
\hline No tags & $95.24 \%$ & $96.33 \%$ & $95.48 \%$ \\
\hline \multicolumn{4}{|c|}{ Table 3. Annotation agreement }
\end{tabular}

Fei Xia et al. Developing Guidelines and Ensuring Consistency for Chinese Text Annotation. 2000.

COLING.

\section{Conclusions}

In this paper, we presented our most up-to-date results on Vietnamese treebank construction. This project is coming to final stage. We continue to annotate more text, revise data by syntactic phenomenon and feedback from users. We also use statistical techniques to analyze treebank data to find out errors and fix them. We intend to publish these data on LDC this year.

\section{Acknowledgments}

This paper is supported by a national project named Building Basic Resources and Tools for Vietnamese Language and Speech Processing, KC01.01/06-10.

\section{Reference}

Diệp Quang Ban. 2005. Ngữ pháp tiếng Việt (2 tập). NXB Giáo duc.

Cao Xuân Hạo. 2006. Tiếng Việt sơ thảo ngữ pháp chức năng. NXB Khoa học Xã họi.

Nguyễn Minh Thuyết và Nguyễn Văn Hiệp. 1999. Thành phần câu tiếng Việt. NXB ĐHQG Hà Nội.

Ủy ban Khoa học Xã hội Việt Nam. 1983. Ngữ pháp tiếng Việt. NXB Khoa học Xã hội.

Adam Berger, Stephen D. Pietra, and Vincent D. Pietra. 1996. A maximum entropy approach to natural language processing. Computational Linguistics, (22-1).

David Chiang and Daniel M. Bikel. 2002. Recovering Latent Information in Treebanks. COLING.

Michael Collins. 1999. Head-Driven Statistical Models for Natural Language Parsing. PhD thesis, University of Pennsylvania.

John Lafferty, Andrew McCallum, and Fernando Pereira. 2001. Conditional random fields: Probabilistic models for segmenting and labeling sequence data. ICML.

Mitchell P. Marcus et al. Building a Large Annotated Corpus of English: The Penn Treebank. 1993. Computational Linguistics.

L. H. Phuong, N. T. M. Huyen, R. Azim, H. T. Vinh. A hybrid approach to word segmentation of Vietnamese texts. Proceedings of the $2^{\text {nd }}$ International Conference on Language and Automata Theory and Applications, Springer LNCS 5196, Tarragona, Spain, 2008.

Anna M.D. Sciullo and Edwin Williams. 1987. On the definition of word. The MIT Press. 